\title{
Comparación in vitro del efecto del pulido en la morfología superficial de tres resinas compuestas.
}

In vitro comparison of the effect of polishing the surface morphology three composites.

Mary Fukuhara-Nakama ${ }^{1, a}$, Martin Quintana-Del Solar ${ }^{l, a}$, Jose Aguilar-Mendoza ${ }^{2, b}$.

\section{RESUMEN}

Objetivos: El objetivo del presente estudio fue evaluar la morfología de las superficie de tres resinas compuestas de restauración directa Filtek Z 250 (3M), TetricCeram (Ivoclar-Vivadent) y Te Econom (Ivoclar-Vivadent) pulidas con el sistema de pulido Astropol (Ivoclar-Vivadent), dada la importancia de obtener superficies altamente estéticas y adecuadamente lisas que dificulten el acúmulo de placa bacteriana, la tinción de la restauración que pueda afectar el color y la salud bucal del paciente. Material y Métodos: Se evaluaron 15 especímenes, cinco de cada resina compuesta las cuales fueron pulidas con el sistema Astropol ${ }^{\circledR}$ para posteriormente obtener las medidas con el microscopio confocal de superficie y tras procesar los datos con el programa ArRUGa 2.0 y utilizar los parámetros utilizados fueron el Ra, RMS, Skewness, Kurtosis, factor de Wenzel y dimensión fractal. Los resultados fueron analizados con las pruebas de ANOVA de una sola vía y de Sheffe con un nivel de confianza del 0.05 , los cuales indicaron que había una diferencia estadísticamente significativa en los parámetros de rugosidad de las tres resinas. Resultados: En el presente estudio se encontró que la superficie más lisa fue la obtenida utilizando la resina Z-250-3M y el sistema de pulido Astropol-IvoclarVivadent. Conclusiones: La utilización de los parámetros de rugosidad de forma y un instrumento como el microscopio confocal nos da mayor información del comportamiento de las rugosidades.

\section{Palabras clave: RESINAS SINTÉTICAS, SÍNTESIS QUÍMICA, IN VITRO.}

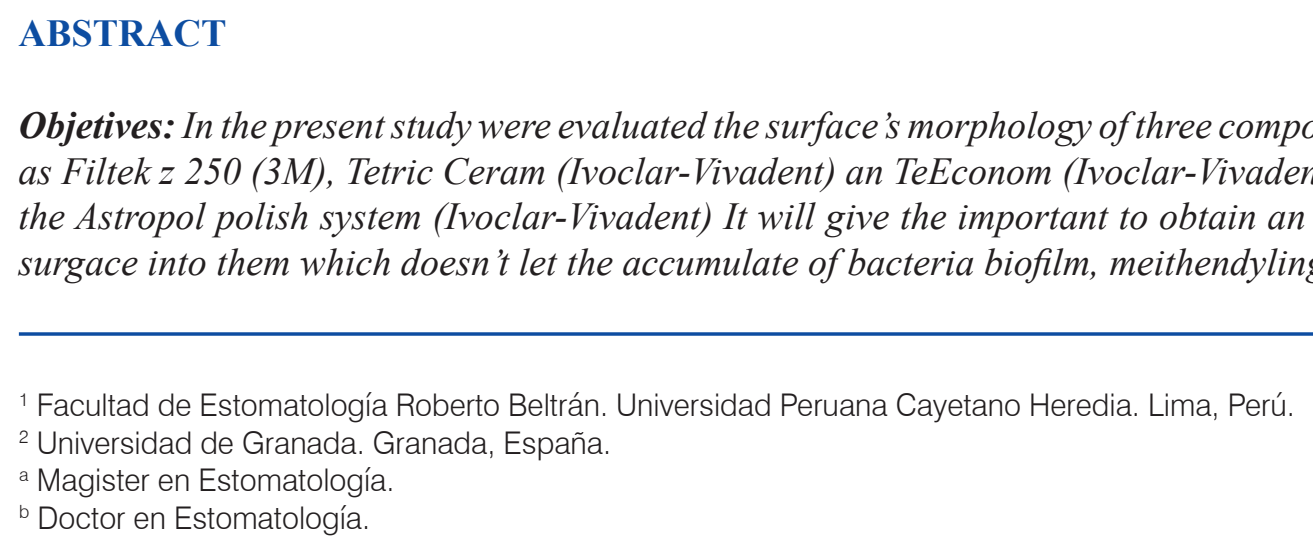

\footnotetext{
${ }^{1}$ Facultad de Estomatología Roberto Beltrán. Universidad Peruana Cayetano Heredia. Lima, Perú.

2 Universidad de Granada. Granada, España.

a Magister en Estomatología.

b Doctor en Estomatología.
}

Objetives: In the present study were evaluated the surface's morphology of three composed resin of direct recovery as Filtek z 250 (3M), Tetric Ceram (Ivoclar-Vivadent) an TeEconom (Ivoclar-Vivadent) They were polished with the Astropol polish system (Ivoclar-Vivadent) It will give the important to obtain an adequate high esthetic flat surgace into them which doesn't let the accumulate of bacteria biofilm, meithendyling on the recovery, so that it 
can to change the color and the patient's oral health. Material and Methods: They were evaluated 15 samples, five of each composed resin, so we proceed to polish the surfaces with Astropol system, for then to obtain the measure in the Confocal Microscope, the dates were proced throught ArRuga 2.0 program and use roughness parameters. The parameters used were Ra, RMS, Skewness, kurtosis, Wenzel factor and Fractal dimention, the results were analyzed by the Anova Test on one way and the Sheffe's test, with the confidence's level of 0.05 those indicated that there were a significant stadistict difference into the winkled state's parameter of the three resin. Results: In the present study we found that followed the most flat surface was obtained throught of the used Filtek Z-250-3M resin and the Astropol-IvoclarVivadent polish system of this was obtained a good polish. Conclusions: We found is too important to obtained a good polish, so we found is too important parameter, of form and an instrument like Confocal microscope, it describes us the best conduct of the winkled states.

\section{Keywords: RESINS, SYNTHETIC, CHEMICALSYNTHESIS, IN VITRO.}

\section{INTRODUCCIÓN}

Las restauraciones dentales realizadas con resinas compuestas deben poseer una superficie lisa, para evitar el acúmulo de placa bacteriana, lo cual influye en la duración del material restaurador y en la estabilidad del color, así como en el estado gingival de la zona circundante a la misma $(1,2,3)$.

El desarrollo de los materiales de restauración directa con características de color del diente para poder satisfacer las actuales exigencias estéticas de los pacientes, hizo necesaria la introducción de diferentes resinas compuestas entre ellas están las resinas compuestas tales como Filtek Z250-3M.U.S.A, TetricCeram y Te-EconomIvoclarVivadent-.Liechtenstein, esta última de muy bajo costo y posible alternativa para nuestra realidad.

Existen diversos métodos usados para evaluar el pulido de las restauraciones como el uso de microscopio de barrido, el microscopio óptico, la perfilometría, la cual es usada debido a su sencillez y sobretodo objetividad. Los parámetros más utilizados son: el valor promedio de rugosidad $(\mathrm{Ra})$, la distancia entre el punto más alto y el más bajo del registro (Rt), se define como la desviación absoluta del promedio de las irregularidades rugosas de la línea media en una longitud, la desviación estándar de la distribución de las alturas (RMS), de las cuales, en este estudio se utilizará el Ra y el Rms como parámetros de altura, además se evaluará el parámetro de Skweness ( Rsk ) que es la medida de rugosidad próxima a la línea central y el parámetro de kurtosis ( Rku) que es la medida de rugosidad en la extensión de la curva de densidad del perfil rugosidad como parámetros de forma, así mismo se incluirá el factor de Wenzel y dimensión fractal que son parámetros muy usados en física, que describe mejor el comportamiento de la rugosidad.

Podemos justificar este trabajo por la necesidad de mayor información de las resinas compuestas y en especial del Te-Econom (IvoclarVivadent) de la cual no se tiene datos en la literatura, además incluir nuevos parámetros para la evaluación de las superficies rugosas.

Stoddard y col. sugieren el uso de instrumentos abrasivos de grano fino de carburo de silicio o con base de óxido de aluminio o diamante con el fin de logra rugosidades de mínimas dimensiones al efectuar el pulido de la superficie de la resina (4).

Los principios de pulido de resinas compuestas no son diferentes del pulido de metales. La secuencia del pulido con abrasivos, va desde el abrasivo más grueso hasta el más fino, se considera que los cauchos para acabado de resina compuestas (Astropol-VivadentIvoclar) son un tipo de puntas abrasivas, las mismas están presentes en diferentes formas y tamaños para individualización del acabado para las diferentes restauraciones y/o para cada segmento de una restauración, éstos se componen de caucho, carburo de silicio, óxido de aluminio, dióxido de titanio y óxido férrico, además de contener polvo de diamante.

La resistencia a la abrasión de la matriz resinosa es menor que aquella del relleno y es esta diferencia la causa de la dificultad de acabado y pulido de las resinas compuestas, durante el pulido la fase orgánica se abrasiona más rápido que la fase de relleno y en 
consecuencia se vuelve rugosa $(4,5)$.

El presente estudio permitió conocer la topografía de las resinas Filtek Z250®, TetricCeram ${ }^{\circledR}$ y T-econom ${ }^{\circledR}$ pulidas con el sistema Astropol (Ivoclar-Vivadent) utilizando un perfilómetro del tipo confocal, utilizando parámetros de rugosidad que describen tanto la altura como la forma y el comportamiento real de la rugosidad (6).

\section{MATERIAL Y MÉTODOS}

Se utilizaron tres grupos de estudio, uno por cada material, cada grupo estuvo formado de 15 medidas, obtenidas de las 5 cavidades ( 3 medidas de cada cavidad), todos los especimenes fueron pulidos con la misma técnica y con el sistema de pulido Astropol (Ivoclar-vivadent) (Fig 1).

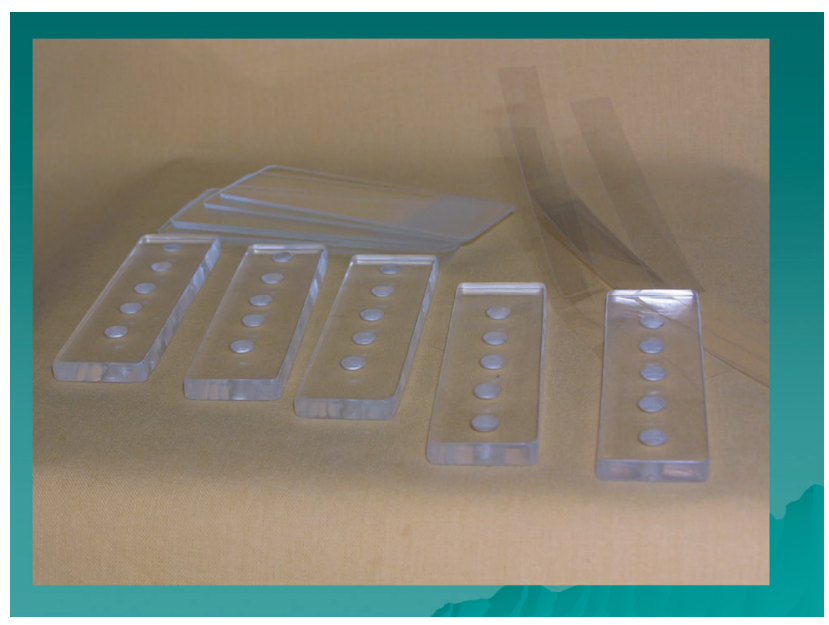

Fig 1. Láminas de acrílico utilizadas para las muestras del estudio

Fueron fabricadas 5 especímenes para cada grupo experimental confeccionadas como sigue: Se colocaron cada tipo de resina compuesta en cinco cavidades cilíndricas hechas en láminas de acrílicos con una profundidad de $3 \mathrm{~mm}$ de diámetro y $5 \mathrm{~mm}$ de profundidad. Las resinas fueron manipuladas según las instrucciones del fabricante, posteriormente los especimenes fueron cubiertos con una cinta de celuloide y una lámina portaobjetos, para luego ser polimerizados por 60 segundos. En cada uno de los especimenes se procedió a efectuar el pulido, este procedimiento fue realizado en la Universidad Peruana Cayetano Heredia.

El pulido se realizó con el sistema de Astropol a 10
000 rpm, con agua en aerosol, se realizó puliendo secuencialmente con los cauchos para acabado, pulido y pulido de alto brillo, por 30 segundos cada caucho, dichas muestras fueron enviadas al laboratorio de Física Aplicada de la Facultad de Ciencias de la Universidad de Granada, para la obtención de medidas, y posteriormente los datos fueron enviados a la Universidad Peruana Cayetano Heredia para ser procesados.

\section{Obtención de medidas}

El microscopio confocal de superficie es de Sensofar y usa el software PLu con el cual se evaluó las superficies de las resinas de nuestro estudio, fueron observadas a 100X y una resolución de imagen de $762 \mathrm{x}$ 560 píxeles, el área evaluada fue de 138,88 x 102,02 um las topografías fueron obtenidas con los siguientes parámetros $\mathrm{Z}$ de 16 simétrico, con una resolución de 0,2 um en altura y en de 0,18 um de lateral $(X, Y)$.

El motor mueve la mesa portamuestra y se desplaza para realizar un barrido simétrico cada $0,2 \mathrm{um}$ y en cada plano escanea 426,72 puntos de los que solo son captados los que se encuentren enfocados, al terminar el barrido total de toda la distancia en $\mathrm{Z}$ establecido el software PLu ajusta a un algoritmo $30 \%$ mejor que el microscopio láser confocal, con este algoritmo de mayor contraste obtenemos la medida de la superficie logrando visualizarlo en 2D o en 3D.

Los medidas guardadas en formatos PLu propios del microscopio confocalSensofar del cual se extrajeron archivos de datos de las medidas o posiciones $(\mathrm{x}, \mathrm{y}, \mathrm{z})$ de cada píxel del área de 138,8 x 102,02 um seleccionada para nuestro estudio.

\section{Tratamiento de los datos con ArRUGa 2.0}

Los archivos de datos extraídos del confocal fueron importados dentro del programa ArRUGa 2.0 desarrollado por el Dr. Miguel A. Rodríguez Valverde (7), para obtener los parámetros de rugosidad simples como Ra, RMS, PV, además de los otros parámetros de forma como Skewness, Kurtosis, el otro parámetro innovador importante para nuestros estudios es el factor de wenzel, y otro parámetro que es la dimensión fractal estos pueden describir o caracterizar mejor la topografía superficial.

Otra herramienta que presenta este programa del área medida y visualizaciones en dos dimensiones $\mathrm{y}$ en tres dimensiones. 


\section{Plan de análisis}

Para el análisis estadístico se utilizó la prueba de ANOVA de una sola vía y la prueba de Sheffé con un nivel de confiabilidad del 95\%, para evaluar si existe diferencia significativa en la topografía superficial entre las resinas compuestas en cada parámetro de la rugosidad.

\section{RESULTADOS}

Los datos obtenidos fueron analizados con los test de ANOVA de una sola vía, de Scheffe's con un nivel de significancia de 0,05 .

A pesar de que las tres resinas compuestas luego de ser pulidas podían observarse relativamente lisas, al evaluar el promedio del Ra de cada una de ellas que se muestra en la tabla 1 y en el gráfico 1 , puede observarse que la superficie de la resina Filtek Z-250 (3M) pulida con el sistema Astropol (Ivoclar-Vivadent) es presenta menor rugosidad en comparación con la resina Tetricceram y el Teconom, en orden creciente de rugosidad.

Esta diferencia en las superficies de las tres resinas compuestas al ser analizadas estadísticamente, se concluye que esta diferencia es estadísticamente significativa.
En la tabla 1 y el gráfico 2, puede observarse los valores del RMS, y se puede apreciar con mayor claridad la diferencia en el grado de rugosidad de las superficies de las tres resinas compuestas, apreciándose que para la resina compuesta Filtek z 250 se ha encontrado un valor de 0,47 , que es menor al de la resina TetricCeram que posee un RMS de 1,64 y en tercer lugar la resina Te Econom con un RMS de 2,28.

En cuanto al parámetro de kurtosis para las tres resinas compuestas, puede observarse en la tabla 1 y el gráfico 4 que la resinas Filtek Z 250, el TetricCeram y el Te Econom se observa una kurtosis mayor a 3, ( $4,88,3,84$ y 3,32 respectivamente).

En la tabla 1 y el gráfico 5 se observan los datos obtenidos para el factor de Wenzel de cada resina compuesta, siendo mayor para la resina Filtek Z 250 y menor para el Teconom, estadísticamente se obtuvo que la diferencia no es estadísticamente significativa.

Con respecto al parámetro de dimensión Fractal, se han obtenido resultados similares, cuyo valor es de dos como puede observarse en la tabla $\mathrm{N}^{\circ} 1$ y en el gráfico $N^{\circ} 6$ observándose que la desviación estándar en el caso de la resina Filtek Z 250 es menor entre las tres $(0,001)$, seguida por Te Econom con una desviación estándar de 0.002 y el TetricCeram cuya desviación es de 0,004 .

Tabla 1. Comparación de los parámetros de rugosidad de las tres resinas compuestas

\begin{tabular}{llll}
\hline & Filtek z 250 & Tetricceram & TeEconom \\
\hline Ra & $0,37(0,29) \mathrm{a}$ & $1,36(0,96) \mathrm{b}$ & $1,92 \quad(0,69) \mathrm{c}$ \\
RMS & $0,47(0,37) \mathrm{a}$ & $1,64(1,14) \mathrm{b}$ & $2,28(0,79) \mathrm{c}$ \\
Skewness & $-0,21(0,77)$ & $-0,19(0,67)$ & $-0,085(0,31)$ \\
kurtosis & $4,88(2,94)$ & $3,84(4,86)$ & $3,32(0,39)$ \\
Factor de Wenzel & $1,02(0,01) \mathrm{a}$ & $1,01(0,01) \mathrm{a}$ & $1,01(0,02) \mathrm{a}$ \\
Dimensión Fractal & $2.000(0.001) \mathrm{a}$ & $2.000(0.004) \mathrm{a}$ & $2.000(0,002) \mathrm{a}$ \\
\hline
\end{tabular}

Los valores promedio en micrómetros de 5 medidas por cada material y la desviación estándar entre paréntesis de cada resina compuesta. 


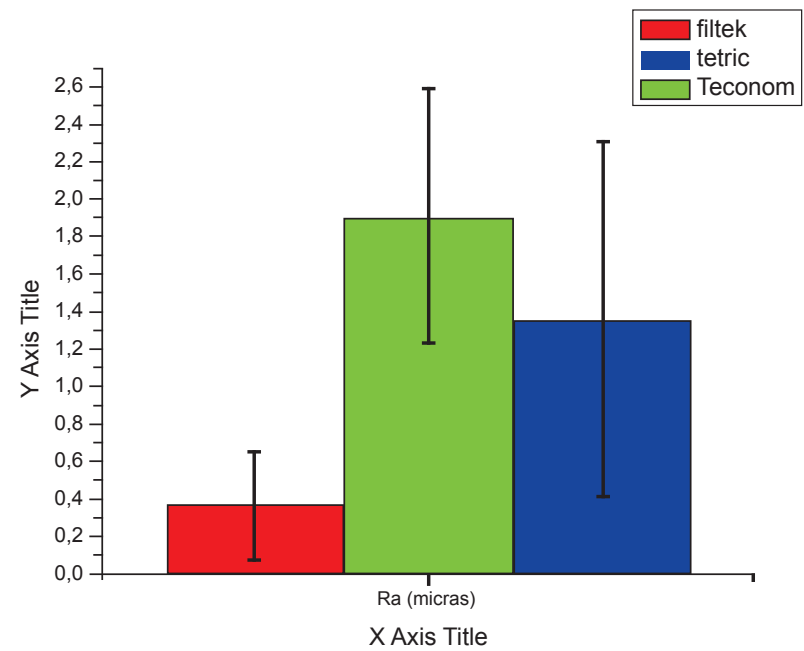

Gráfico 1. Promedio aritmético de la altura de la rugosidad (Ra) de las resinas Filtek z 250, Tetricceram y Te Econom.

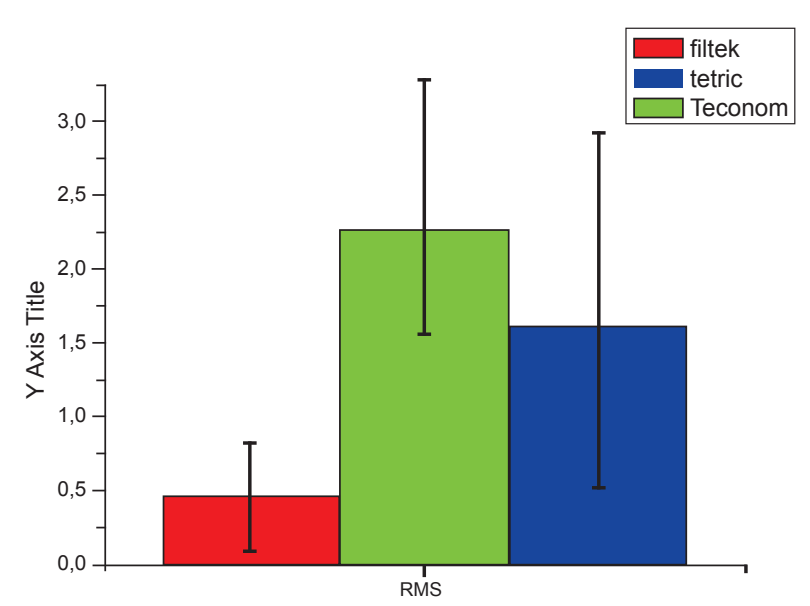

Gráfico 2. Desviación estándar de la distribución de las alturas(RMS) de las resina compuestas Filtek z 250, Tetric ceram y Te Econom.

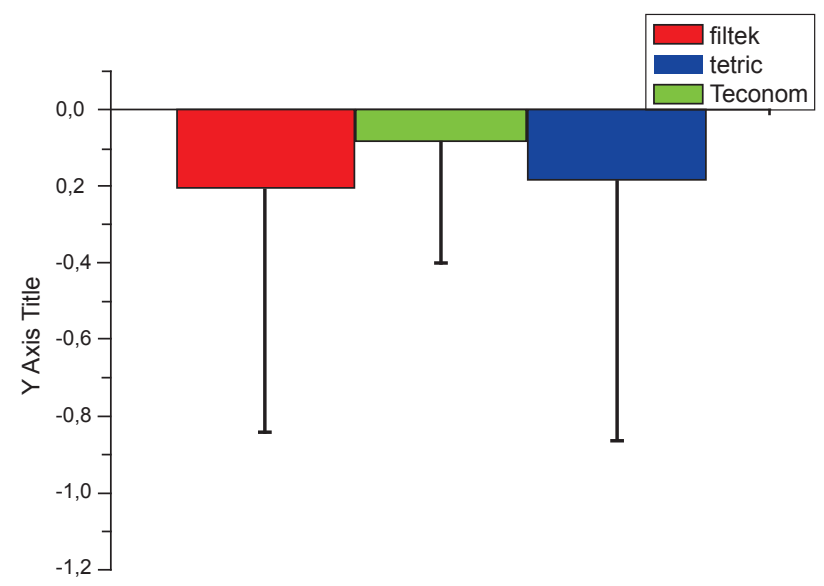

Gráfico 3. Skewness (Rsk) de la resina compuesta Filtek z 250, Tetric Ceram y Te Econom.

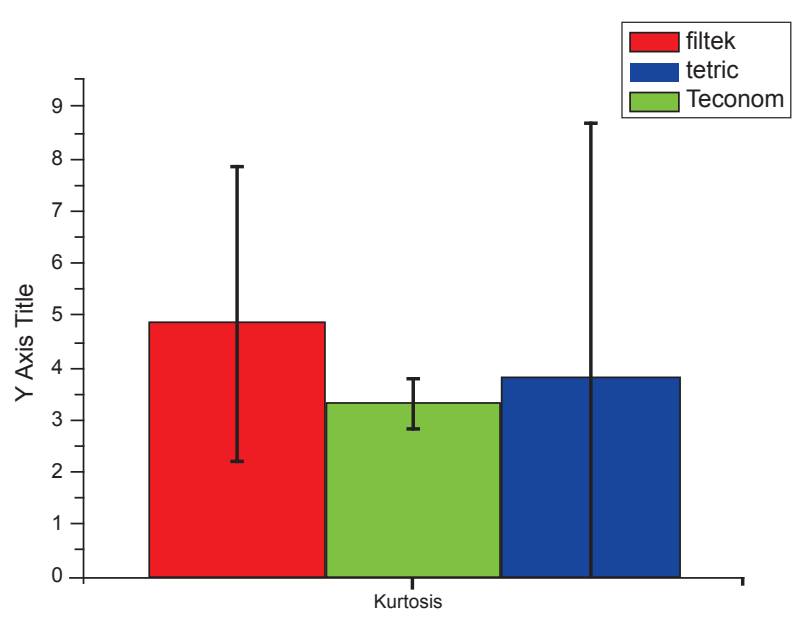

Gráfico 4. Kurtosis de las resinas compuestas Filtek Z 250, Tetric ceram y Te Econom (Rku).

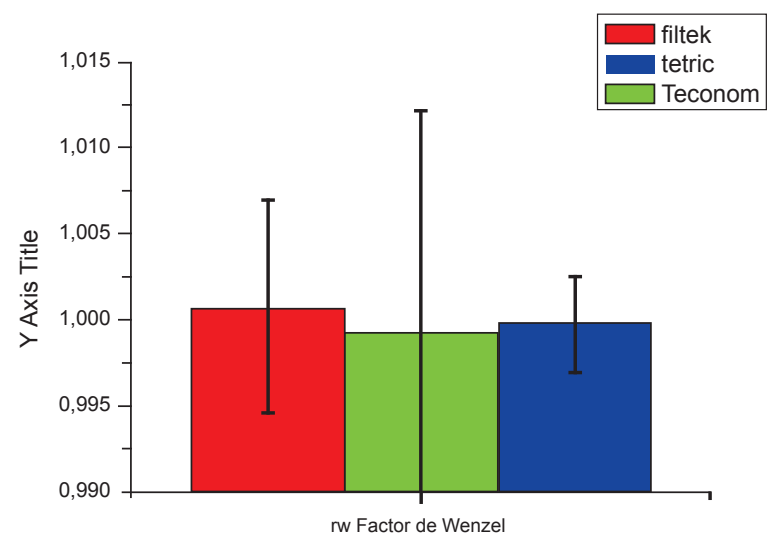

Gráfico 5. Factor de Wenzel para las resinas compuestas Filtek Z 250, Tetric Ceram y Te Econom.

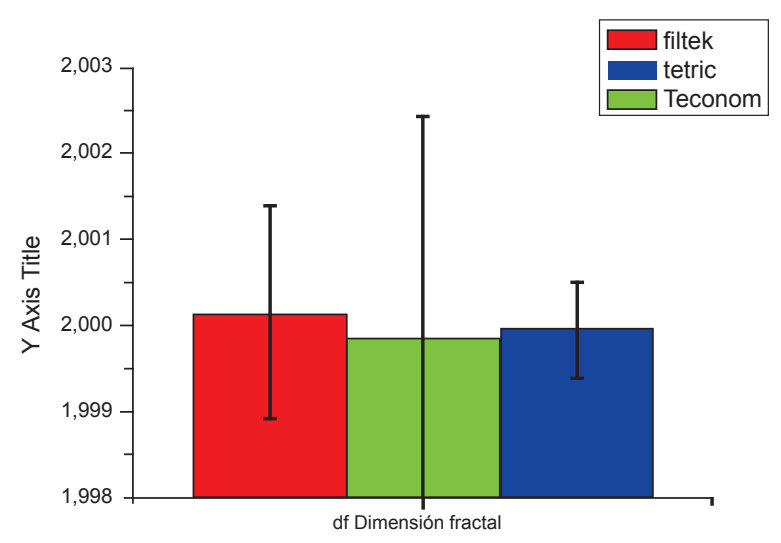

Gráfico 6. Dimensión fractal de las resinas compuestas Filtek $Z$ 250, Tetric Ceram y Te Econom. 


\section{DISCUSIÓN}

Las superficies de las restauraciones de resinas compuestas influyen sobre la calidad y longevidad de las restauraciones dentro de la cavidad oral y la morfología superficial toma cada vez más importancia en las restauraciones actuales debido a las mayores exigencias estéticas de los pacientes y menor tiempo requerido por los operadores, para satisfacer estos requerimientos se modifican las resinas compuestas y tratan de simplificar los sistemas de pulido.

En estudios el uso de la cinta celuloide como técnica para obtener superficies lisas en forma rápida se presenta como desventaja principal que la capa superficial queda formada solo con matriz, la cual se desgasta muy fácilmente, debido a la proporción muy grande de matriz/relleno, haciéndola muy débil, además de la dificultad del uso de la cinta de celuloide en algunas zonas de la boca $(7,8)$. La superficie menos rugosa se obtiene con la cinta de celuloide, y concuerda con muchos otros estudios $(9,12)$.

Como se sabe, existen muchos sistemas para el pulido de las resinas compuestas (12), Joniot y Grégoire y Col encontraron en su estudio que se obtenía una superficie mejor pulida con el uso de fresas de diamante y copas de silicona (9), Berástegui, Brau y Col. encontró mejor resultado con discos de óxido de aluminio, combinado con otro sistema de pulido según el tipo de resina (13). En el presente estudio se utilizó el sistema Astropol (IvoclarVivadent), debido a que es un sistema que ofrece resultados adecuados en el pulido de resinas compuestas, además en la revisión bibliográfica no se ha encontrado estudios similares con este sistema de pulido, el Astropol-IvoclarVivadent, está compuesto por caucho, carburo de silicio, óxido de aluminio, dióxido de titanio y óxido férrico, además de contener polvo de diamante este último busca pulir el relleno de las resinas compuestas. Este sistema tiene pocos colores de cauchos simplificando así a los anteriores sistemas de pulido de múltiples componentes o múltiples pasos. Los resultados obtenidos para las tres resinas compuestas observadas macroscópicamente son muy buenos en cuanto a lisura y estética, los resultados de los parámetros de rugosidad obtenidos en este estudio son semejantes a estudios similares con diferentes tipos de sistemas de pulido $(4,7,11,13,14,15)$.
Las superficies analizadas muestran que el grado de rugosidad dejado por el sistema de pulido Astropol (Ivoclar-Vivadent) depende del tamaño de partícula de la resina.

En el presente estudio, se mostraron diferencias significativas en cuanto a la rugosidad de la superficie de las tres resinas compuestas pulidas por el sistema Astropol-IvoclarVivadent, teniendo en cuenta los parámetros de Ra, RMS, como en otros estudios, la resina compuesta con partículas de relleno más pequeñas presentaron la superficie más lisa.

En el presente estudio se observó que la resina Filtek Z 250-3M (0.001 -3,5 micras), presenta la superficie más lisa luego de ser pulida, seguida del Tetricceram -IvoclarVivadent(0.04-3 micras ) y la resina Teconom-IvoclarVivadent (0,04-7 micras), lo que concuerda con los estudios obtenido por Berástegui y col. en cuanto a que la composición de la resina compuesta tiene un efecto directo en los resultados del pulido de las mismas, así como una resina cuyas partículas de relleno son menores, presentaran luego del pulido superficie más lisa (14). La limitación de Ra y RMS por ser parámetros lineales no dan idea de la superficie y se limita a líneas independientes de la superficie.

James W. Stoddad y col. en su estudio encontraron que al ser pulidas las superficies de las resinas compuestas, las partículas de relleno son removidas causando rugosidades en la superficie, la cual depende del tamaño de las mismas (4), Jonioty col. Y Hoelscher y col concluyen que la rugosidad de la superficie puede determinarse por las características del sistema de pulido y por las propiedades de las resina compuesta $(10,11)$, dentro de estas características considera el tamaño de la partícula de relleno. Ryba y col encontraron que la resina compuesta Filtek Z 250-3M es más fácil de pulir y tiene baja susceptibilidad de teñirse (13).

En el presente estudio, se evalúa la morfología de la superficie de tres resinas compuestas pulidas con el sistema Astropol (Ivoclar-Vivadent), esta evaluación se realizó mediante el uso de la perfilometría, y a diferencia de la mayoría de estudios que utilizan el Ra $(12,18)$ introduce otros parámetros como, el Skwesness, Kurtosis, factor de Wenzel y dimensión fractal. 
Estos parámetros complementan la información limitada que se obtiene con el Ra, además de ser poco sensible para algunas rugosidades. En nuestro estudio muestran la gran relación con el tamaño de partículas de las diferentes resinas evaluadas discriminándolas entre ellas.

Marigo y Col. (19) en un estudio similar en el cual utilizaron parámetros como Ra, RMS, PV, Rsk (Skwesness) y Rku (Kurtosis), observaron en los dos tipos de resina compuesta obtuvo Skwesness negativo igual que el encontrado en nuestro estudio, lo cual indica que luego del pulido se observa mesetas con depresiones proporcionales al tamaño de las particulas, en la superficie propias de un material compuesto y los valores tienen relación con el tamaño de partículas de cada resina compuesta.

Además, analizando el parámetro de Kurtosis mayor a 3, estas son superficies con distribución "leptokurtosis" lo cual significa que las rugosidades tienen mayor altura de pico y valles bajos con una proporción relacionada al tipo de resina, este resultado coincide con el estudio de Marigo y Col. (19), en resinas compuestas utilizado instrumentos muy distintos a los tradicionales como rugosimetros de contacto.

Los otros parámetros de rugosidad como factor de Wenzel y dimension fractal fueron medidos e introducidos en este estudio, para poder obtener valores numéricos comparativos consecuencia de análisis matemáticos de los datos obtenidos con el microscopio Confocal, estos valores nos dan idea cuantitativa de los plano que puede estar las superficies vistas tridimensionalmente y el no tener diferencia entre las resinas compuestas confirman que el material es muy parecido y genera una superficie semejante evaluando tridimensionalmente, y la otra posibilidad es que el sistema de pulido Astropol-IvoclarVivadent, pueda lograr un pulido muy parecido sobre tres resinas diferentes.

En el presente estudio se utilizó el microscopio confocal de luz blanca, el cual tiene muchas ventajas con respecto a la microscopía tradicional, es una microscopia capaz de ofrecer información topográfica de la superficie, en tres dimensiones, dando medidas cualitativa y cuantitativas, además puede reconstruir una imagen tridimensional de la muestra en forma no invasiva, con lo cual se puede reproducirse secuencias clínicas, siendo los resultados obtenidos más fiables. Este instrumento usa su software Plu propio, con el que se evaluó las superficies de las resinas del estudio, adicionalmente, para este estudio se construyó y fue utilizado el programa ArRUGa 2.0, para obtener todos los parámetros de rugosidad de este estudio en especial el factor de Wenzel y dimensión fractal únicos parámetros que pueden acercarnos a medidas tridimensionales de la superficie posiblemente generada por la cinta celuloide y que el relleno es tan pequeño que no afecta a las medidas en las dimensiones medidas cuya resolución se limita a los 200 nanómetros nos quedaría la posibilidad de evaluar con otros instrumentos de mayor resolución como el AFM.

Marigoy col utilizaron un instrumento similar, Perfilómetro ZYGO 3D (ZIGO New View 5000, Lot Oriel, Milano, Italia) pero no utilizaron factor de Wenzel ni dimension fractal, a diferencia de este estudio (19).

El Microscopio Confocal es un instrumento muy útil para muchas investigaciones debido a su versatilidad en la obtención de datos; en nuestro estudio los nuevos parámetros de forma confirman un comportamiento similar de las resinas compuestas ante un tipo de desgaste o pulido, pero la contribución de nuestro estudio es la verificación o confirmación numérica de un fenómeno real, visto con mucha frecuencia en nuestra clínica.

Una de las debilidades observadas en este estudio, fue la realización del pulido en forma manual, ya que la diferencia de presión ejercida al momento del pulido, podría introducir una variación a los resultados.

\section{CORRESPONDENCIA}

Mary FukuharaNakama

Av. Honorio Delgado 430

San Martín de Porres, Lima-Perú

Correo electrónico:

mary.fukuhara@upch.pe

\section{REFERENCIAS BIBLIOGRÁFICAS}

1. Jefferies SR. The art and science of abrasive finishing and polishing in restorative. Dentistry Dent Clin N Am. 1998;42(4):613-27. 
2. Bollen CML, Lambrechts P, Quirynen M. Comparison of surface roughness of oral hard materials to the threshold surface roughness for bacterial plaque retention: a review of the literature. Dent Mater. 1997;(13):258-69.

3. Biedma MB, Rodríguez-Ponce A, López-Campos A, Lois-Mastach F, Pazos-Sierra R, Súarez J. Alteraciones en la densidad óptica de una resina compuesta sometida a la acción de un colorante. Avances en Odontoestomatología. 2000;16(8):479-83.

4. Stoddard J, Johnson G. An evaluation of polishing agents for composite resin. J Prosthet Dent. 1991;65:491-5.

5. Libermand R, Geiger S. Surface texture evaluation of glass ionomer restorative materials polished utilizing poly(acrylic acid) gel. J Oral Rehab. 1994;(21): 87-94.

6. Aguilar J. Caracterización superficial y evaluación de la eficacia de los adhesivos auto grabadores en dentina. Tesis Doctoral. Granada, España.Universidad de Granada, 2004.

7. Passetti A, Cervini M, Guerra M, Staffolani N. Analisis de resinas compuestas en el microscopio electrónico de Barrido (MEB). J de Clínica en Odontología. 1998;14(4):1999.

8. Wilson F, Heath H, Watts DC. Finishing composite restaurative materials. J Oral Rehab 1990; 17: 79-87

9. Ozgunaltay G, Yazic AR, Borucu J. Effect of finishing and polishing procedures on the surface roughness of new tooth coloures restoratives. J Oral Rehab. 2003; 30(2):218.

10.Joniot SB, Gregoire GL, Auther AM, Roques YM. Three dimensional optical profilometry analysis of surface states obtaines after finishing sequences for three composite resins. Operative Dentistry 2000;25:311-5.
11.Park S, Krejcr I, Lutz F. Hardness of celluloid strip finished of polished composite surfaces with time. J. Prosthetic Dentistry. 2000;83:660-3.

12.Hoelscher D, Neme A, Pink F, Hughes P. The effect of three finishing systems on four esthetic restorative materials. Operative Dentistry. 1998;23:36-42.

13.Ryba T, Dunn W, Murchison D. Surface roughness of various packable composites. Operative Dentistry. 2002;27:243-7.

14.Berastegui E, Brau E, Miquel C. Surface roughness of finished composite resins. J. Prosthet Dent. 1992; 68:742-9.

15.Reis A, Giannini M, Lovadino J, Ambrosano G. Effects of various finishing systems on the surface roughness and staining susceptibility of packable composite resins. Dental Material. 2003;19:12-8.

16.Neme A, Franzier K, Roeder L, Debner T. Effect of prophylactic polishing protocols on the surface roughness of esthetic restorative material. Operative Dentistry 2002;27:50-8.

17. Chain M, Baratieri L. Restauraciones estéticas con resinas compuestas en dientes posteriores. 1ra edición. Sao Paulo: Artes Medicom; 2001.

18. Roeder L, Tate W, Powers J. Effect of finishing and polishing procedures on the surface roughness of packable composites. Operative Dentistry. 2000;25:534-43.

19.Marigo L, Rizzi M, La Torre G, Rumi G. 3-D surface profile analysis:Different finishing methods for resins composites. Operative Dentistry. 2001;26:562-8.

Recibido : 29-05-2013

Aceptado: 02-09-2013 